\title{
正癸烷与二甲苯在超临界压力下的热裂解
}

\author{
周 影 ${ }^{1}$ 毛 佳 ${ }^{1}$ 汪必耀 ${ }^{1}$ 朱 权 ${ }^{1}$ 王健礼 ${ }^{2, *}$ 李象远 ${ }^{1, *}$ \\ ( 1 四川大学化学工程学院, 成都 610065; ${ }^{2}$ 四川大学化学学院, 成都 610064)
}

\begin{abstract}
摘要: 采用连续流动装置对正癸烷和二甲苯在超临界压力下的热裂解对比研究. 用气相色谱和色质联用仪对 其气相产物和液相产物进行分析, 计算气相产物产率和裂解转化率, 并运用计算化学方法获得正癸烷和二甲苯 不同化学键的键能, 从实验和理论上分析其裂解反应的难易程度和裂解规律. 实验结果表明, 在 $4 \mathrm{MPa}$ 和 650 、 $700 、 750{ }^{\circ} \mathrm{C}$ 条件下, 正癸烷比二甲苯更容易裂解, 正癸烷裂解产物以 $\mathrm{C}_{1}-\mathrm{C}_{3}$ 小分子的烃类和氢气为主, 而二甲 苯裂解产物主要为乙苯、甲苯和其它芳香类化合物; 键能计算结果表明, 正癸烷碳链骨架的 $C-C$ 键能和 $C-H$ 键能均较小, 裂解反应的诱发步骤应该是 $\mathrm{C}-\mathrm{C}$ 键断裂, 而二甲苯苯环上 $\mathrm{C}-\mathrm{C}$ 和 $\mathrm{C}-\mathrm{H}$ 键能均较大, 裂解诱发 步骤应该是侧链甲基脱氢反应. 因此正癸烷裂解反应以 $\mathrm{C}-\mathrm{C}$ 键断裂和脱氢反应为主, 二甲苯裂解主要发生侧 链甲基 $\mathrm{C}-\mathrm{C}$ 键断裂和脱氢反应, 而芳环则比较稳定, 理论计算键能分析与裂解实验结果一致.
\end{abstract}

关键词: 正癸烷; 二甲苯; 超临界; 热裂解; 键能 中图分类号: 0643

\section{Pyrolysis of $n$-Decane and Dimethylbenzene under Supercritical Pressure}

\author{
ZHOU Hao ${ }^{1} \quad$ MAO Jia ${ }^{1} \quad$ WANG Bi-Yao ${ }^{1} \quad$ ZHU Quan ${ }^{1} \quad$ WANG Jian-Li $\mathrm{Li}^{2, *} \quad$ LI Xiang-Yuan ${ }^{1, *}$ \\ $\left({ }^{1}\right.$ College of Chemical Engineering, Sichuan University, Chengdu 610065, P. R. China; \\ ${ }^{2}$ College of Chemistry, Sichuan University, Chengdu 610064, P. R. China)
}

\begin{abstract}
The pyrolysis of $n$-decane and dimethylbenzene under supercritical pressure was studied using a continuous flow reactor. Samples were heated to a temperature of 650,700 , or $750{ }^{\circ} \mathrm{C}$ under a pressure of $4 \mathrm{MPa}$ without oxygen. $n$-Decane pyrolyzed more easily than dimethylbenzene. We analyzed gaseous products by online gas chromatography, and liquid products by gas chromatography-mass spectrometry, allowing us to calculate the cracking gas yield and cracking conversion of these systems. $\mathrm{A}$ quantum chemistry computation was used to evaluate the binding energies of $\mathrm{C}-\mathrm{C}$ and $\mathrm{C}-\mathrm{H}$ bonds in $n$-decane and dimethylbenzene. Both experimental and theoretical results were also used to analyze the cracking reactivity of these species. Analysis of the components in the products indicated that the main products of $n$-decane were $\mathrm{C}_{1}-\mathrm{C}_{3}$ hydrocarbons and hydrogen, whereas ethylbenzene, toluene and other aromatic compounds were the main products of dimethylbezene after pyrolysis. Binding energy calculations showed that both $\mathrm{C}-\mathrm{C}$ and $\mathrm{C}-\mathrm{H}$ bonds in $n$-decane possessed lower binding energies than those in dimethylbezene, and a $\mathrm{C}-\mathrm{C}$ bond was the weakest. In dimethylbenzene, a $\mathrm{C}-\mathrm{H}$ bond in the methyl groups was the weakest, and its binding energy was much smaller than those of the $\mathrm{C}-\mathrm{C}$ and $\mathrm{C}-$ $\mathrm{H}$ bonds in the benzene ring. Therefore, the main reactions in the cracking process of $n$-decane are breakage of a $\mathrm{C}-\mathrm{C}$ bond and dehydrogenation. However, the cracking process in dimethylbenzene mainly involves the fracture and dehydrogenation of methyl groups. The theoretical calculations reasonably explained the experimental phenomena.
\end{abstract}

Received: November 15, 2012; Revised: February 5, 2013; Published on Web: February 6, 2013.

"Corresponding authors. WANG Jian-Li, Email: wangjianli@scu.edu.cn; Tel: +86-28-85402951.

LI Xiang-Yuan, Email: xyli@scu.edu.cn; Tel: +86-28-85405233.

The project was supported by the National Natural Science Foundation of China (91116001/A0204).

国家自然科学基金(91116001/A0204)资助项目

C Editorial office of Acta Physico-Chimica Sinica 
Key Words: $n$-Decane; Dimethylbenzene; Supercritical condition; Pyrolysis; Binding energy

\section{1 引 言}

吸热型碳氢燃料主要是利用燃料在较高温度 下发生裂解反应, 生成一些高热值的小分子烃类和 氢气以供燃烧, 并在此过程中吸收大量无用以致有 害的能量来达到冷却发动机的目的. 此类燃料组分 非常复杂, 主要包括链烷烃、环烷烃、芳香烃和烯 烃, 为了提高裂解反应的转化率和选择性, 需要更 好地了解各种组分裂解的详细过程, 对反应的过程 和规律进行深入的研究, 从而为改善然料的性能以 及提高燃料吸热能力提供更好的依据.

正癸烷是具有代表性的直链烷烃, 常作为替代 组分研究航空煤油的特性. ${ }^{1,2}$ 目前对其裂解燃烧的 研究在理论上已经比较成熟, ${ }^{3-7}$ Zeppieri 等 ${ }^{8}$ 还根据 正庚烷机理模型发展了正癸烷的部分简化的骨架 机理, 但在实验上的研究则很少, ${ }^{9-11}$ 大部分都集中 在催化裂解方面, 而对正癸烷本身的热裂解规律研 究的不多. 芳香烃具有高能量密度, ${ }^{12,13}$ 是航空燃料 的重要组分, 而且苯等芳香烃一直被视为结焦的前 驱体, ${ }^{14}$ 但是在实验和理论上对芳香烃裂解规律的 研究均很少. ${ }^{15-17}$ 此外, 比较芳香烃和直链烷烃在相 同条件下的热裂解规律, 能从实验上探究这两种不 同的化学结构的裂解差异.

燃料作为冷却剂时, 需要在高于临界压力下实 现等密度相变, 避免燃料 “沸腾” 对发动机工作的稳 定性造成不利影响. 正癸烷和二甲苯的临界压力分 别为 2.11 和 $3.54 \mathrm{MPa}$, 因此本文在 $4 \mathrm{MPa}, 650 、 700$ 、 $750{ }^{\circ} \mathrm{C}$ 条件下开展这两种燃料成分的热裂解对比研
究, 着重考察这两种物质在气相产物产率、转化率, 气相产物和液相产物成分上的差异, 并从理论上对 反应规律进行分析和验证.

\section{2 实 验}

\section{1 实验原料和实验装置}

本实验选用纯度 $\geq 99.9 \%$ 的正癸烷和二甲苯作 为实验原料. 实验装置如图 1 所示, 包括进料系统、 反应系统、冷凝系统和产物分析系统. 反应管为 2 $\mathrm{mm}$ 内径的 304 不锈钢管, 反应管加热段长 $700 \mathrm{~mm}$. 用高压平流泵将反应原料以 $1.0 \mathrm{~g} \cdot \mathrm{s}^{-1}$ 的质量流量注 入反应器进行热裂解反应, 系统压力用背压阀调 节, 反应产物通过冷凝器后到达气液分离器, 收集 液相产物和气相产物, 并用气相色谱对气相产物进 行在线分析. 为了实现在超临界压力下的裂解, 本 文控制系统压力为 $4 \mathrm{MPa}$, 产物出口温度为 650 、 $700 、 750{ }^{\circ} \mathrm{C}$, 反应前用 $99.99 \%$ 的氮气吹扫反应管.

\section{2 分析方法}

气相产物用上海计算技术研究所的 GC-2000III 型气相色谱仪进行分析, 小分子烃类采用 $\mathrm{HP}-\mathrm{Al} / \mathrm{S}$ 毛 细管柱 $(50 \mathrm{~m} \times 0.530 \mathrm{~mm} \times 15.0 \mu \mathrm{m}$, Agilent 公司 $)$ 和氢 火焰离子化检测器; 氢气采用自制的 $2 \mathrm{~m}$ 填充柱(固 定相为 TDX-101)和热导检测器. 载气为高纯氮气.

液相产物用美国 Agilent 公司的 HP-6890/5973 型气相色谱/质谱联用仪进行分析, 色谱柱为 HP$5 \mathrm{MS}$ 毛细管分离柱 $(30 \mathrm{~m} \times 25 \mathrm{~mm} \times 0.25 \mu \mathrm{m}$, Agilent 公司), 载气为高纯氮气.

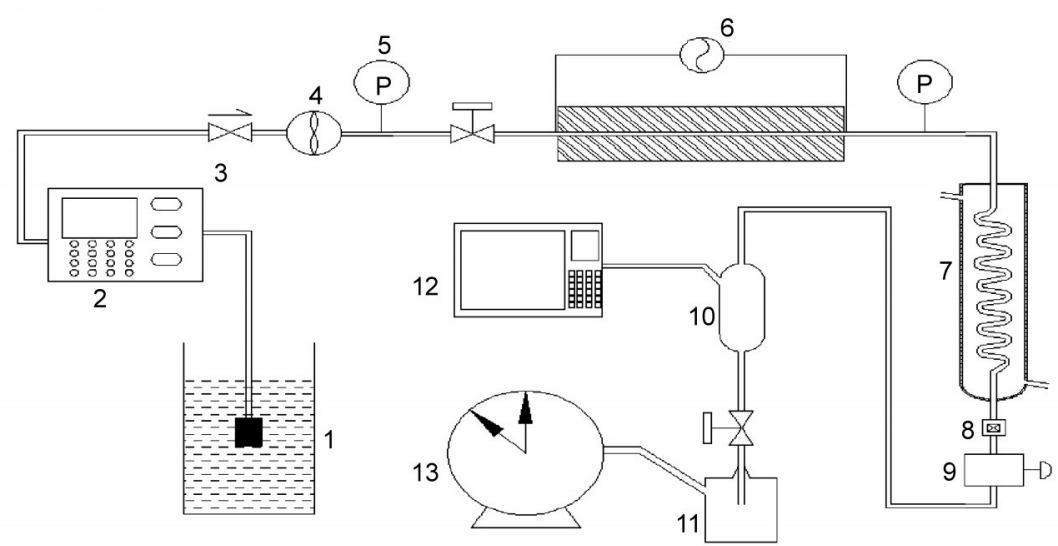

图 1 超临界裂解反应装置图

Fig.1 Setting drawing of supercritical pyrolysis

1-feed stock, 2-pump, 3-check valve, 4-mass flowmeter, 5-manometer, 6-electrical heater, 7-condenser, 8-filter, 9-back pressure controller, 10-seperator, 11-liquid collector, 12-gas chromatography, 13-gas flowmeter 


\section{3 实验结果与分析}

\section{1 气相产物产率和转化率}

正癸烷和二甲苯裂解气相产物产率 $(y)$ 由(1)式 计算, 裂解转化率 $(x)$ 由(2)式计算:

$$
\begin{aligned}
& y=m_{\mathrm{g}} / m \\
& x=1-m_{1} w_{1} / m
\end{aligned}
$$

其中, $m$ 为单位时间原料总进料质量, $m_{\mathrm{g}}$ 为单位时间 裂解气相产物质量, $m_{1}$ 为单位时间裂解液相产物质 量, $w_{1}$ 为裂解液相产物中残存原料的质量分数. 图 2 显示了正癸烷和二甲苯裂解气相产物产率. 由图 2 可以看出, 随着温度升高, 正癸烷的气相产物产率 明显增大, 远大于二甲苯的值. 说明正癸烷的裂解 生成了大量的小分子烃类, 并且随着温度的升高裂 解程度加剧, 而二甲苯则不易生成小分子烃类.

表 1 列出了正癸烷和二甲苯的裂解转化率. 从 表 1 可以看出, 二甲苯的转化率在 $750{ }^{\circ} \mathrm{C}$ 时达到了 约 70\%, 说明二甲苯在实验条件下会发生裂解反 应, 但主要途径是生成其它液态烃类产物. 结合其 气相产物产率可以推断二甲苯裂解过程中可能主 要发生侧链甲基断裂和脱氢反应, 苯环结构保持稳 定, ${ }^{18}$ 并主要生成一些其它芳香类化合物. 正癸烷的 转化率和气相产物产率的趋势相一致, 并且转化率 和气相产物产率相差不大, 说明正癸烷裂解过程 中, $\mathrm{C}-\mathrm{C}$ 键和 $\mathrm{C}-\mathrm{H}$ 键断裂, 生成大量的小分子烃 类, 断键程度随温度升高而加深, 同时可能伴有自 由基的聚合反应发生. 二者裂解反应的差异主要是 由正癸烷和二甲苯裂解反应活化能的不同造成的. 正癸烷裂解反应的诱发步骤, 应该是 $\mathrm{C}-\mathrm{C}$ 键断裂, 而这些 $\mathrm{C}-\mathrm{C}$ 单键均由 $\mathrm{C}$ 的 $s p^{3}$ 杂化轨道形成, 键能

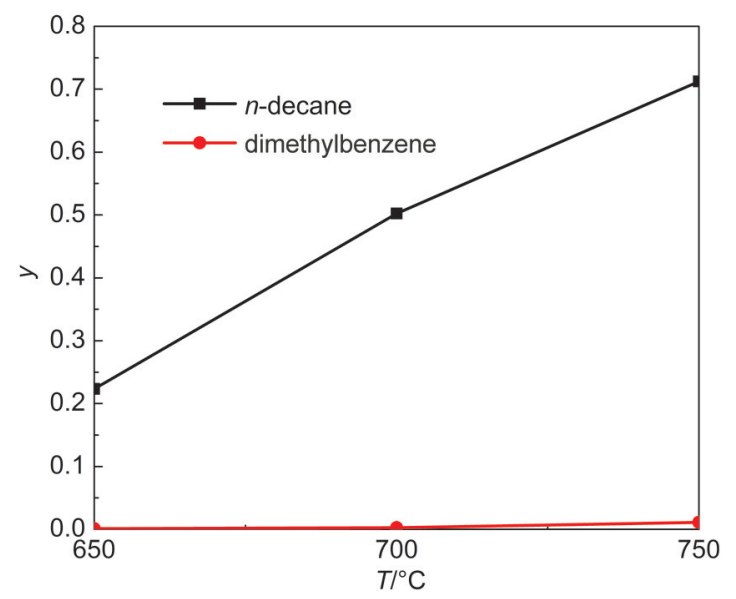

图 2 正癸烷和二甲苯裂解气相产物产率 ( $y$, 质量分数)

Fig.2 Cracking gas yields ( $y$, mass fraction) of $\boldsymbol{n}$-decane and dimethylbenzene
表 1 正癸烷和二甲苯的裂解转化率 ( $x$, 质量分数)

Table 1 Conversion ratio ( $x$, mass fraction) of $n$-decane and dimethylbenzene

\begin{tabular}{ccc}
\hline \multirow{2}{*}{$T /{ }^{\circ} \mathrm{C}$} & \multicolumn{2}{c}{$x / \%$} \\
\cline { 2 - 3 } & $n$-decane & dimethylbenzene \\
\hline 650 & 47.61 & 28.28 \\
700 & 81.40 & 46.23 \\
750 & 93.08 & 70.74 \\
\hline
\end{tabular}

较小. 在二甲苯裂解诱发阶段, 苯环开环时需要破 坏共轭 $\pi$ 键, 活化能较大, 而二甲苯上取代基的 $\mathrm{C}-$ $\mathrm{C}$ 键由苯环 $\mathrm{C}$ 原子的 $s p^{2}$ 杂化轨道和甲基 $\mathrm{C}$ 原子 $s p^{3}$ 杂化轨道组成, 键能比正癸烷的 $\mathrm{C}-\mathrm{C}$ 键能高, 裂解 难度更大.

\section{2 裂解产物分布}

\subsection{1 气相产物}

图 3 为正癸烷裂解气相产物含量随温度的变 化情况. 由图 3 可以看出, 正癸烷裂解气相产物主要 有甲烷、乙烷、乙烯、丙烯和氢气，随着温度的升高 甲烷含量不断上升, 乙烷、乙烯和丙烯含量呈现下 降趋势, 证实正癸烷裂解主要生成 $\mathrm{C}_{1}-\mathrm{C}_{3}$ 的小分子 烃类和氢气, 正癸烷裂解反应以链上 $\mathrm{C}-\mathrm{C}$ 键断裂 和脱氢反应为主, 并且随着温度升高断键程度加 深, 发生二次裂解, 反应向着生成甲烷的方向进行.

图 4 为二甲苯裂解气相产物含量随温度的变化 情况. 由图 4 可以看出, 二甲苯裂解气相产物主要是 甲烷和氢气, 几乎没有其它小分子烃类生成, 结合 气相产物产率和转化率证实二甲苯裂解主要发生 侧链甲基 $\mathrm{C}-\mathrm{C}$ 键断裂和脱氢反应, 苯环基本保持 稳定. 随着温度升高, 甲烷含量降低而氢气含量升 高, 结合转化率可以发现二甲苯裂解生成的甲烷可 能与侧链甲基自由基发生反应生成乙苯等其它芳

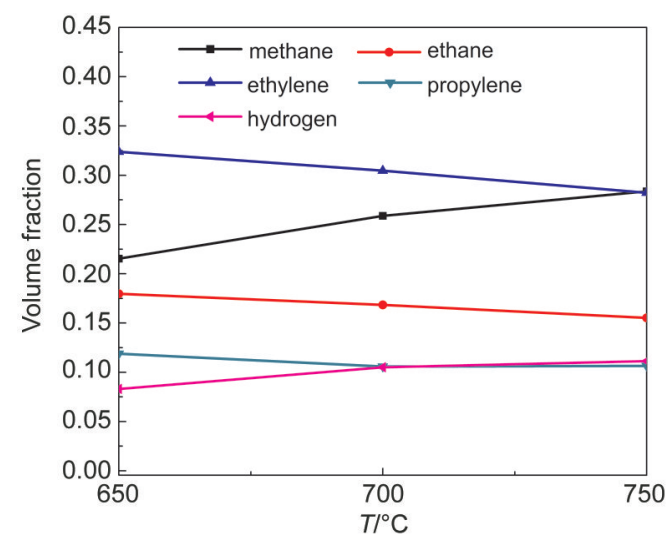

图 3 正癸烷裂解气相产物含量(体积分数)

Fig.3 Gas product contents of $\boldsymbol{n}$-decane cracking (volume fraction) 


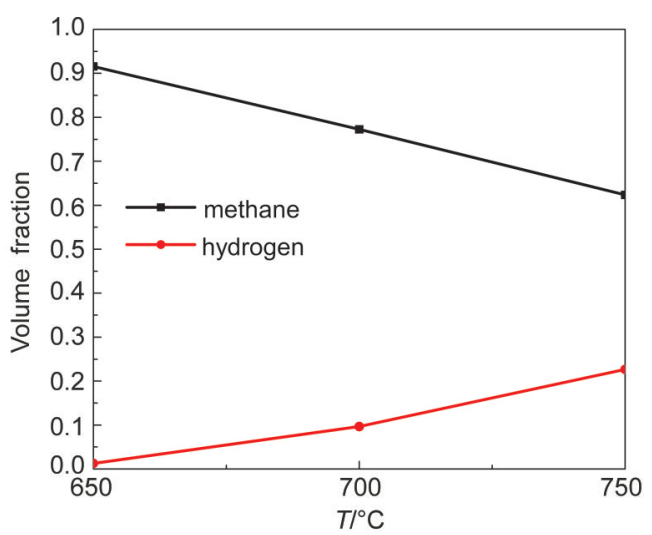

图 4 二甲苯裂解气相产物含量(体积分数)

Fig.4 Gas product contents of dimethylbenzene cracking (volume fraction)

香类化合物, 并且可能有稠环芳烃出现.

\section{2 .2 液相产物}

正癸烷和二甲苯在 $4 \mathrm{MPa}$ 和出口温度为 650 、 $700 、 750{ }^{\circ} \mathrm{C}$ 条件下裂解的液相产物通过色质联用仪 进行分析, 并用峰面积归一化法计算各产物成分的 相对含量(质量). 结果表明正癸烷裂解的液相产物 成分复杂. 我们将其归为链烷烃、环烷烃、链烯烃、 环烯烃和芳香烃五类, 其中链烷烃和链烯烃中产物 $\mathrm{C}$ 原子数均不大于正癸烷; 而二甲苯裂解液相产物 几乎全为芳香类化合物, 主要成分为甲苯、乙苯和 未裂解的二甲苯.

由图 5 可以看出, 在 $650{ }^{\circ} \mathrm{C}$ 正癸烷裂解液相产 物以链烷烃和链烯烃为主, 再次说明正癸烷裂解反 应主要为链上 $\mathrm{C}-\mathrm{C}$ 键断裂和脱氢反应. 同时可以 看出, 随着温度升高, 链烷烃含量降低, 链烯烃含量 先升高后降低, 环烷烃、环烯烃和芳香烃含量升高, 说明随着温度上升, 正癸烷裂解深度逐渐加深, 链

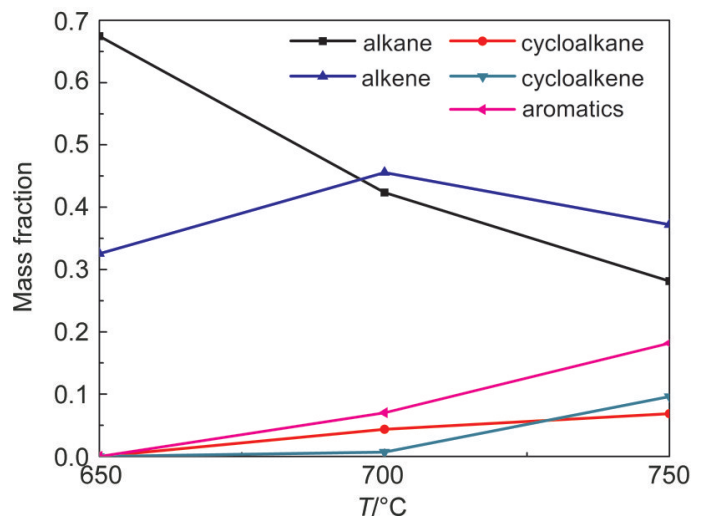

图 5 正癸烷裂解液相产物含量(质量分数)

Fig.5 Liquid product contents of $\boldsymbol{n}$-decane cracking (mass fraction)

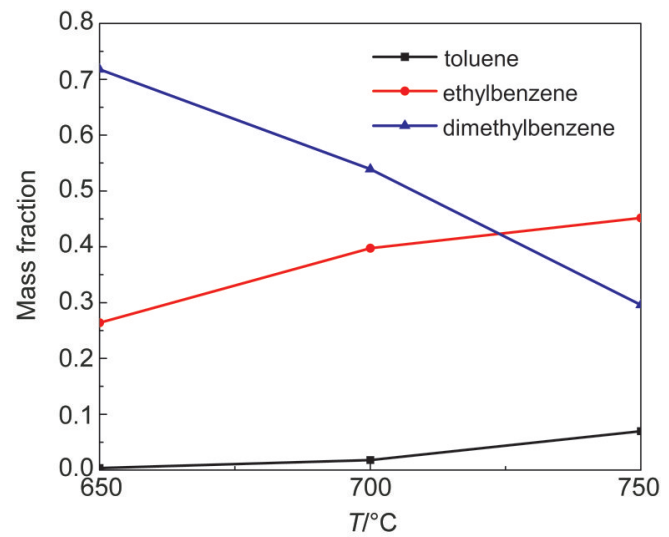

图 6 二甲苯裂解液相产物含量(质量分数)

Fig.6 Liquid product contents of dimethylbenzene cracking (mass fraction)

烷烃脱氢生成链烯烃和环烷烃, 部分链烯烃和环烷 烃再脱氢聚合生成环烯烃和芳香烃, 这与气相产物 中氢气含量不断升高相符合, 并且随着温度升高, 聚合程度加深, 产物越来越复杂, 在 $750{ }^{\circ} \mathrm{C}$ 发现有 稠环芳烃生成.

由图 6 可以看出, 在 $650{ }^{\circ} \mathrm{C}$ 二甲苯裂解液相产 物主要是乙苯和未裂解的二甲苯, 说明二甲苯发生 裂解反应主要是以断侧链甲基和脱氢反应为主, 并 且生成的甲基与苯环上甲基脱氢的自由基结合生 成乙苯, 可能的反应步骤如下:
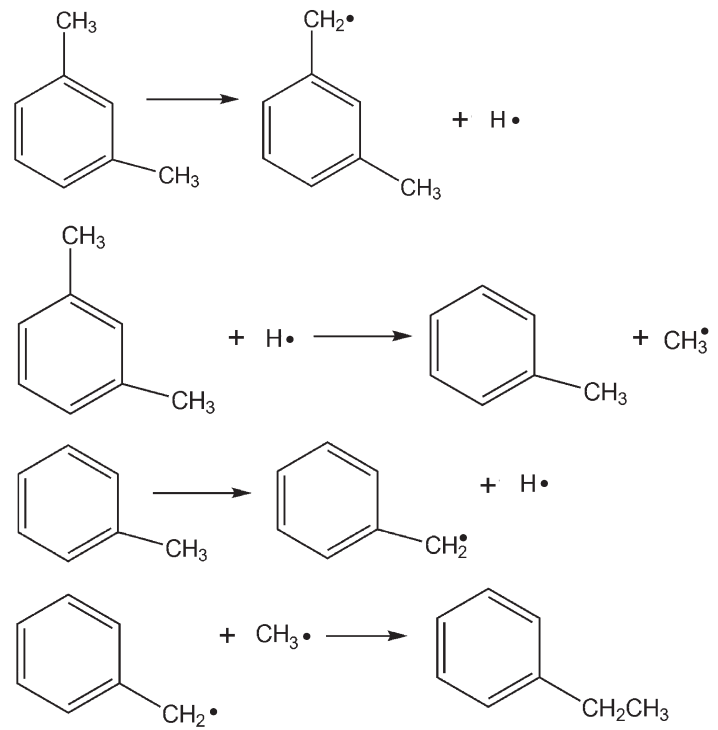

更深入的研究需要构建裂解反应的复杂反应 机理. 可以看出, 随着温度升高, 液相产物中的二甲 苯含量降低, 乙苯和甲苯含量上升, 三种物质总含 量在 $750{ }^{\circ} \mathrm{C}$ 仍占整个液相产物的 $70 \%$ 以上, 其余也 为芳香类化合物, 有稠环芳烃出现, 说明二甲苯的 裂解反应由于受芳环稳定性的影响, 与正癸烷裂解 
反应相比较, 反应程度并不深, 裂解产物也比较单 一, 主要是发生侧链甲基断裂和转移反应, 同时也 有一定程度的聚合反应发生.

为了进一步验证二甲苯裂解过程中苯环的稳 定性, 本文计算了单位时间二甲苯裂解液相产物中 苯环的摩尔含量与原料中苯环的摩尔含量之比, 以 此来说明二甲苯裂解过程苯环的裂解程度. 计算结 果显示, 在 $650 、 700 、 750{ }^{\circ} \mathrm{C}$, 二甲苯裂解液相产物与 原料中含有的苯环摩尔含量之比始终在 $90 \%$ 以上, 分别为 $98.08 \% 、 96.83 \% 、 92.16 \%$, 说明二甲苯裂解开 环比率很低.

\section{4 理论计算}

本文采用密度泛函(DFT)方法在 BHandHLYP/ $6-31+\mathrm{G}(d, p)$ 基组 ${ }^{19,20}$ 水平上对所有反应中涉及到的 物种的几何结构进行优化和频率计算, 并在此基础 上计算了相应键能. 通过计算发现零点能校正对键 能的计算影响较小, 因此本文未考虑零点能校正. 对稳定分子断裂为两个自由基片段情形, 键能 $E_{\mathrm{b}}$ 表 示为:

$$
E_{\mathrm{b}}=E_{\mathrm{x}} \bullet+E_{\mathrm{y}} \bullet-E_{\mathrm{xy}}
$$

其中, $E_{\mathrm{xy}}$ 为反应物分子的能量, $E_{\mathrm{x}}$. 和 $E_{\mathrm{y}}$. 为断键后 的两个自由基片段的能量. 对于苯环开环情形, 键 能 $E_{\mathrm{b}}$ 表示为:

$$
E_{\mathrm{b}}=E \cdot \mathrm{xy} \cdot-E_{\mathrm{xy}}
$$

其中, $E_{\mathrm{xy}}$ 为芳烃分子的能量, $E \cdot \boldsymbol{x}_{\mathrm{x} y}$. 为苯环 $\mathrm{C}-\mathrm{C}$ 键断 裂后的双自由基的能量. 考虑到化学键较强的相互 作用, 本文未考虑基组重叠误差. 本文所有计算均 在 Gaussian 03 程序包 ${ }^{21}$ 上完成. 由于二甲苯与甲苯 的结构非常相似, 仅相差一个甲基, 因此它们相应 的化合键键能也非常接近, 所以本文用甲苯代替二 甲苯计算相应的键能, 键能计算的原子编号见图 7, 计算结果列于表 2 .

由表 2 可见, 正癸烷碳链骨架的 $\mathrm{C}-\mathrm{C}$ 键能比 $\mathrm{C}-\mathrm{H}$ 键能小, 因而裂解的诱发步骤应该是 $\mathrm{C}-\mathrm{C}$ 键 的断裂反应. 而甲苯情形则大不相同, 除苯环上 C$\mathrm{C}$ 键能由于共轭效应而加强外, 苯环上的 $\mathrm{C}-\mathrm{H}$ 键由 于 $\mathrm{C}$ 原子采用 $s p^{2}$ 杂化而比烷烃 $\mathrm{C}-\mathrm{H}$ 键能更大, 相 较于侧链甲基不容易发生拆环和脱氢反应. 在取代 基上, $\mathrm{C}-\mathrm{H}$ 键和苯环上 $\mathrm{C}-\mathrm{H}$ 键相比, 由于 $\mathrm{C}$ 原子 采用 $s p^{3}$ 杂化, 键能降低, 因而取代基上的脱氢反应 难度较小, 而 $\mathrm{C}-\mathrm{C}$ 键键能由于苯环和取代基之间 的 $p \pi$ 共轭效应, 因此键能比普通的 $\mathrm{C}-\mathrm{C}$ 单键键能

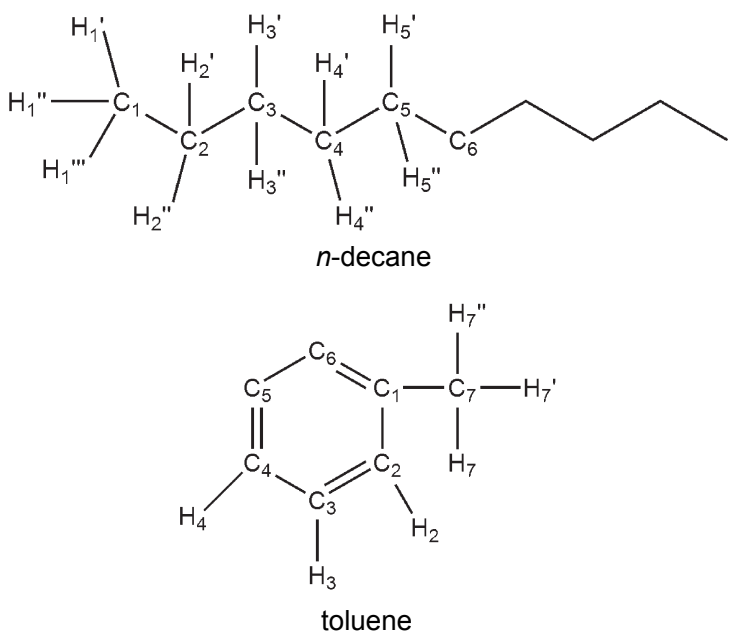

图 7 键能计算原子编号

Fig.7 Atomic numbering in the calculation of binding energy

表 2 正癸烷和甲苯中 $\mathbf{C}-\mathbf{C}$ 和 $\mathbf{C}-\mathbf{H}$ 键能 $\left(\boldsymbol{E}_{\mathrm{b}}\right)$

Table $2 \mathrm{C}-\mathrm{C}$ and $\mathrm{C}-\mathrm{H}$ binding energies $\left(E_{\mathrm{b}}\right)$ in $\boldsymbol{n}$-decane and toluene

\begin{tabular}{cccc}
\hline Bond in $n$-decane & $E_{\mathrm{b}} /\left(\mathrm{kJ} \cdot \mathrm{mol}^{-1}\right)$ & Bond in toluene & $E_{\mathrm{b}} /\left(\mathrm{kJ} \cdot \mathrm{mol}^{-1}\right)$ \\
\hline $\mathrm{C}_{1}-\mathrm{C}_{2}$ & 343.13 & $\mathrm{C}_{1}-\mathrm{C}_{7}$ & 400.58 \\
$\mathrm{C}_{2}-\mathrm{C}_{3}$ & 329.22 & $\mathrm{C}_{1}-\mathrm{C}_{2}$ & 696.70 \\
$\mathrm{C}_{3}-\mathrm{C}_{4}$ & 330.96 & $\mathrm{C}_{2}-\mathrm{C}_{3}$ & 568.55 \\
$\mathrm{C}_{4}-\mathrm{C}_{5}$ & 331.81 & $\mathrm{C}_{3}-\mathrm{C}_{4}$ & 568.39 \\
$\mathrm{C}_{5}-\mathrm{C}_{6}$ & 331.65 & $\mathrm{C}_{7}-\mathrm{H}_{7}$ & 364.42 \\
$\mathrm{C}_{1}-\mathrm{H}_{1}$ & 416.72 & $\mathrm{C}_{2}-\mathrm{H}_{2}$ & 461.79 \\
$\mathrm{C}_{2}-\mathrm{H}_{2}$ & 399.80 & $\mathrm{C}_{3}-\mathrm{H}_{3}$ & 462.53 \\
$\mathrm{C}_{3}-\mathrm{H}_{3}$ & 400.45 & $\mathrm{C}_{4}-\mathrm{H}_{4}$ & 464.78 \\
$\mathrm{C}_{4}-\mathrm{H}_{4}$ & 400.16 & & \\
$\mathrm{C}_{5}-\mathrm{H}_{5}$ & 400.06 & & \\
\hline
\end{tabular}

大. 可以判断, 甲苯裂解的诱发步骤应该是取代基 上的 $\mathrm{C}-\mathrm{H}$ 键断裂, 但这个步骤比正癸烷的 $\mathrm{C}-\mathrm{C}$ 键 断裂仍然要困难得多. 通过表中数据分析, 从理论 上可以预测正癸烷比二甲苯更容易发生裂解反应, 并且正癸烷裂解反应主要为链上 $\mathrm{C}-\mathrm{C}$ 键的断裂及 后续的脱氢反应, 而二甲苯裂解反应主要为甲基脱 氢和进一步的取代基分离, 苯环基本保持相对稳 定. 键能的计算结果很好地解释了两种单组分燃料 的裂解实验现象.

\section{5 结 论}

本文通过两种单组分燃料的热裂解比较, 发现 了正癸烷和二甲苯裂解性能的明显差异. 实验结果 表明, 和二甲苯相比, 正癸烷裂解容易得多, 正癸烷 裂解反应过程中 $\mathrm{C}-\mathrm{C}$ 键的断裂和脱氢反应可以在 任何位置发生, 而二甲苯裂解反应则以取代基上的 $\mathrm{C}-\mathrm{C}$ 键的断裂和脱氢反应为主, 在本文的实验温 
度下, 难以发生开环反应。

作为代表性的链烷烃, 正癸烷的裂解产物主要 为 $\mathrm{C}_{1}-\mathrm{C}_{3}$ 小分子的烃类和氢气, 并且随着温度升高 反应向着生成甲烷和氢气的方向进行, 同时, 其液 相产物成分复杂, 随着温度升高环烃和芳香烃含量 上升, 脱氢聚合程度加深.

二甲苯是一种重要的芳香烃, 其裂解产物以乙 苯、甲苯和其它芳香类化合物为主, 并且随着温度 升高乙苯和甲苯含量上升, 也有一定程度的聚合反 应发生, 有稠环芳烃生成, 同时, 其气相产物含量很 低, 主要为甲烷和氢气.

通过两种体系键能的对比计算表明, 正癸烷各 个化学键键能均较小, 裂解反应的诱发步骤应该是 $\mathrm{C}-\mathrm{C}$ 键断裂, 与此不同, 二甲苯苯环上 $\mathrm{C}-\mathrm{C}$ 和 $\mathrm{C}-$ $\mathrm{H}$ 键能均较大, 不易发生开环和环上脱氢反应, 裂解 诱发步骤应该是侧链甲基脱氢反应, 以及更高温度 下取代基的分离和苯环缩合, 进而形成稠环芳烃. 理论计算很好地解释了实验现象.

\section{References}

(1) Dagaut, P.; Bakali, E. A.; Ristori, A. Fuel 2006, 85, 944. doi: 10.1016/j.fuel.2005.10.008

(2) Humer, S.; Frassoldati, A.; Granata, S.; Faravelli, T.; Ranzi, E.; Seiser, R.; Seshadri, K. Proc. Combust. Inst. 2007, 31, 393. doi: 10.1016/j.proci.2006.08.008

(3) Westbrook, C. K.; Pitz, W. J.; Herbinet, O.; Curran, H. J.; Silke, E. J. Combust. Flame 2009, 156, 181. doi: 10.1016/ j.combustflame.2008.07.014

(4) Olchanski, E.; Burcat, A. Int. J. Chem. Kinet. 2006, 38, 703.

(5) Bikas, G.; Peters, N. Combust. Flame 2001, 126, 1456. doi: 10.1016/S0010-2180(01)00254-1

(6) Nehse, M.; Warnatz, J.; Chevalier, C. Symposium (International) on Combustion 1996, 26, 773. doi: 10.1016/S0082-0784(96) 80286-4

(7) Battin-Leclerc, F.; Fournet, R.; Glaude, P. A.; Judenherc, B.; Warth, V.; Come, G. M.; Scacchi, G. Proc. Combust. Inst. 2000,
28, 1597. doi: 10.1016/S0082-0784(00)80557-3

(8) Zeppieri, S. P.; Klotz, S. D.; Dryer, F. L. Proc. Combust. Inst. 2000, 28, 1587. doi: 10.1016/S0082-0784(00)80556-1

(9) Yin, K. L.; Wu, G. Y.; Chen, Z. L. Acta Petr. Sin. 2006, 17, 77. [殷开梁, 邬国英, 陈正隆. 石油学报, 2006, 17, 77.]

(10) Wang, Z. W.; Zhang, X. W.; Mi, Z. T.; Hao, W. H. Petrochem. Technol. 2005, 34, 518. [王占卫, 张香文, 米镇涛, 郝伟华. 石油化工, 2005, 34, 518.]

(11) Jiao, Y.; Li, J.; Wang, J. B.; Wang, J. L.; Zhu, Q.; Chen, Y. Q.; Li, X. Y. Acta Phys. -Chim. Sin. 2011, 27, 1061. [焦 毅, 李 军, 王静波, 王健礼, 朱 权, 陈耀强, 李象远. 物理化学 学报, 2011, 27, 1061.] doi: 10.3866/PKU.WHXB20110437

(12) Pitz, W. J.; Cernansky, N. P.; Dryer, F. L.; Egolfopoulos, F. N.; Farrell, J. T.; Friend, D. G.; Pitsch, H. In SAE 2007 Transactions Journal of Passenger Cars-Mechanical Systems, SEA Paper 2007-01-0175, 2007.

(13) Farrell, J. T.; Cernansky, N. P.; Dryer, F. L.; Friend, D. G.; Hergart, C. A.; Law, C. K.; McDavid, R. M.; Mueller, C. J.; Patel, A. K.; Pitsch, H. In 2007 SAE International Congress, SEA Paper 2007-01-0201, Detroit, MI, 2007.

(14) Sivaramakrishnan, R.; Tranter, R. S.; Brezinsky, K. J. Phys. Chem. A 2006, 110, 9388. doi: 10.1021/jp060820j

(15) Zhang, L. D.; Cai, J. H.; Zhang, T. C.; Qi, F. Combust. Flame 2010, 157, 1686. doi: 10.1016/j.combustflame.2010.04.002

(16) Lannuzel, F.; Bounaceur, R.; Michels, R.; Scacchi, G.; Marquaire, P. J. Anal. Appl. Pyrolysis 2010, 87, 236. doi: 10.1016/j.jaap.2010.01.001

(17) Richter, H.; Howard, J. B. Prog. Energy Combust. Sci. 2000, 26 , 565. doi: 10.1016/S0360-1285(00)00009-5

(18) Guo, L. Z. Study on Stability and Endothermic Capability of Hydrocarbon Fuels. MS Dissertation, Tianjin University, Tianjin, 2005. [郭兰珍. 碳氢化合物安定性和吸热性能的 研究[D]. 天津: 天津大学, 2005.]

(19) Perdew, J. P.; Burke, K.; Ernzerhof, M. Phys. Rev. Lett. 1996, 77, 3865. doi: 10.1103/PhysRevLett.77.3865

(20) Becke, A. D. J. Chem. Phys. 1993, 98, 5648. doi: 10.1063/ 1.464913

(21) Frisch, M. J.; Trucks, G. W.; Schlegel, H. B.; et al. Gaussian 03 [CP]; Gaussian Inc.: Pittsburgh, PA, 2003. 